\title{
Clinical trials of contraceptive agents in those under 16 years of age: Are they necessary, ethical or legal?
}

\author{
Joe Brierley, Vic Larcher
}

\section{INTRODUCTION}

Children and young people need safe and effective medicines that reflect their changing physiological emotional and psychological status. Ethical and legal tensions arise in the testing of medicines, especially those that are connected with sexual health and sensitive questions of behaviour and lifestyle. Young people's freedom to explore their developing sexuality and independence has to be set against the need to protect them from abuse and exploitation, and the associated risks of pregnancy and sexually transmitted diseases.

Safe and effective contraception for young people under 16 years of age is considered an important part of the overall campaign to reduce teenage pregnancies. However, these agents are not well tolerated in this age group, and there has been limited research to address this. Despite recent welcomed clinical trials of investigational medicinal products (CTIMP) applications to research ethics committees (RECs) in the UK, concern

Paediatric Bioethics, Great Ormond St Hospital, London, UK

Correspondence to Dr Joe Brierley, Department of Paediatric Intensive care, Great Ormond Street Hospital for Children NHS Trust, Great Ormond St, London WC1N 3JH, UK; joe.brierley@gosh.nhs.uk has been raised about the ethical and legal situation surrounding the proposed inclusion into such studies of those as young as 12 years of age.

Research protocols for CTIMPs must conform to regulatory and legal frameworks and be reviewed and approved by RECs before proceeding. ${ }^{12}$ Little formal guidance exists as to how RECs might address proposals to conduct safety and efficacy trials of contraceptive agents in young people under the age of 16 .

The purpose of this paper is to consider the ethical and legal issues posed by such proposals and suggest how guidance might be framed.

\section{BACKGROUND}

There are strong socio-biological and demographic reasons to consider the development of contraceptive agents for children under the age of 16 years.

Despite a recent fall in conception rates in 13-15 year olds, the UK still has the second highest teenage pregnancy rate in the developed world. ${ }^{34}$ Although 70\%-75\% of pregnancies in this age group occur in 15 year olds, over 1000 pregnancies per annum occur in younger individuals, ${ }^{3}$ and pregnancy has been reported in 12 year olds. ${ }^{5}$

Both the age of menarche (mean 12.7 years; range $7-17)^{6}$ and that of first sexual experience (median 16 years) ${ }^{7}$ have fallen over time. In the last UK national survey of sexual attitudes and lifestyle, up to $40 \%$ of 15 year olds had some form of sexual experience ${ }^{8}$ and one-in-five 13 year olds stated that they had taken part in oral or penetrative sex. It is accepted that younger individuals tend to use less effective methods of contraception, for example, condoms or methods that are reliable in older women, less effectively-for example, oral contraceptive agents. ${ }^{4}$ Suggestions that $10 \%-15 \%$ of adolescents on hormonal contraception still get pregnant are logical corollaries of the high incidence of reported side effects, especially breakthrough bleeding, experienced in younger women, with consequently high discontinuation rates in the younger age groups. ${ }^{9}$ There is less experience with the use of potentially more effective methods, for example, long-acting reversible contraceptives (LARCs) and perhaps concomitant reluctance to use them in this age group. ${ }^{1011}$

Despite this, recent applications to undertake CTIMPS of contraceptive agents in UK children as young as 12 years of age have highlighted both the ethical and the legal issues involved and the practical implications for RECs, researchers and the children and their families themselves.

These concerns aside, health risks and adverse socio-economic consequences provide strong imperatives to reduce teenage pregnancy rates. Educational interventions ${ }^{12}$ the growth of abstinence programmes, ${ }^{13}$ attitudinal shifts towards continuing education and the perceived stigmatisation of teenage parenthood may have played a role in the reduction of teenage pregnancy rates in the USA and the UK. ${ }^{14}$ Despite this, the need for safe, effective contraceptive agents remains. 


\section{Ethical considerations}

Adults with capacity are free to make their own choices about contraception. The importance of this freedom has been regarded as sufficient to overcome moral and religious objections to contraception.

Young people, who are perceived as lacking capacity and being vulnerable to coercive influences, may not be granted this freedom. But if they can demonstrate sufficient capacity to make free choices over sexual activity and relationships, and do understand the implications for themselves and their families, denial of access to contraception advice and treatment seems manifestly unjust. Regardless of capacity, young people should have access to appropriate information, for example, by education about relationships and sexual health, which will enable them to exercise future choice. It can be argued that society should not intervene in intensely personal choices made by those with capacity, irrespective of age, unless it is to prevent harm to others. But society does restrict some choices by age, for example, marriage, because of the seriousness of the decision or because it has legitimate interests in the outcome.

Even if there are concerns that young people may be unable to exercise free choices over conception, there is still an obligation to protect young people against the risk of pregnancy that contraception may avert. Easy access to contraception neither necessarily increases the risk of sexual activity nor the harms associated with it, for example, sexually transmitted diseases. ${ }^{15}$ Indeed, the provision of confidential contraceptive advice and treatment provides an opportunity for education and personal discussion of sexual health matters that is consistent with professionals' duty of care to their patients.

Overall, given the same objectives of preventing unwanted pregnancy, the use of contraceptive agents, especially those that prevent ovulation, fertilisation or implantation, appear to engender less sustainable moral objections than those produced by termination. Contraception produces less physical and psychosocial harms than termination of pregnancy, an important consideration given that approximately $60 \%$ of pregnancies in children under the age of 16 years are terminated.

\section{Legal considerations}

In the UK, individuals under the age of 16 can receive contraceptive advice and treatment without the knowledge or consent of their parents, provided they

have the capacity to consent. In England and Wales, this is defined as the ability to understand fully the nature and purpose of what is proposed and its implications for themselves and their family (Gillick competence). ${ }^{16}$ Professionals should satisfy themselves that the young person fulfils Fraser's guidelines for provision of contraception (box 1). In Scotland, a young person's capacity to consent to medical treatment is determined by the professional providing the treatment. ${ }^{17}$

In both jurisdictions, young people are owed the same duty of confidentiality as adults. Confidentiality may only be breached when the health, safety and welfare of the young person or others would be at grave risk, for example, from abuse or exploitation. $^{18}$

Most jurisdictions have legislation intended to protect young people from abuse and exploitation that may accompany some forms of adolescent sexual behaviours. In England and Wales, the age of consent for sexual activity (of whatever orientation) as specified in the Sexual Offences Act (SOA) 2003 is 16 years. ${ }^{19}$ While sexual activity below this age is technically a criminal offence, the seriousness with which it is regarded depends on the ages of those involved and whether there is evidence of-or good reason to suspect-abuse, exploitation or coercion. Prosecution of young people of similar age and understanding, and who engage in mutually consensual sexual activity is not usually regarded as being in the public interest. However, children under the age

Box 1 The 'Fraser Guidelines' state that all the following requirements should be fulfilled

- The young person will understand the professional's advice.

- The young person cannot be persuaded to inform their parents.

- The young person is likely to begin, or to continue having, sexual intercourse with or without contraceptive treatment.

- Unless the young person receives contraceptive treatment, their physical or mental health, or both, are likely to suffer.

- The young person's best interests require them to receive contraceptive advice or treatment with or without parental consent.

Gillick v. West Norfolk and Wisbech Area Health Authority (1985) of 13 years of age are regarded as lacking the capacity to give valid consent to sexual activity and such activity is a serious offence. ${ }^{19}$

Although it is an offence under the SOA to aid, abet, counsel or otherwise facilitate sexual offences in children under the age of 16 years, professionals are specifically permitted to give contraceptive advice or treatment intended to prevent pregnancy or promote the child's physical or emotional well-being, though a lower age limit is not specified. ${ }^{19} 20$

Under the Clinical Trial Regulations 2004, a minor, for CTIMP purposes, is anyone under the age of 16 years. $^{21}$ The preamble to the European Clinical Trials Directive (EC2001/20), ${ }^{22}$ which the Clinical Trial Regulations transposed into UK law, set out fundamental ethical principles. It helpfully contrasted the usual restrictive concept of only including children in CTIMPs when there are grounds to expect administration of the IMP to be of direct benefit to them, thereby outweighing risks, with the overwhelming need for research in children per se. Medicinal products likely to be of significant value for children and young people must be fully studied. So, the case that contraceptive investigational medicinal products (IMPs) ought to be tested in young people in whom they are likely to be used, and helpful, for example, those between 13 and 16 years of age, seems likely to be lawful under the European directive, but for younger age groups this might well not be the case.

\section{Practical implications}

Existing guidance on the provision of contraceptive services for young people from menarche onwards have specified that age alone should not limit contraceptive choices provided medical eligibility criteria are met. ${ }^{2324}$ Most emphasise the requirement to provide young people with sufficient information on contraceptive methods to enable them to make choices that suit their needs and preferences. 52325 However, there are little trial data on which to base specific recommendations about safe and effective contraception for young people in the UK. This is particularly so with respect to LARCs, ${ }^{20} 26$ although they as a class may have properties that make them more suitable for young people than alternatives currently used and are more frequently used in Europe. $^{11}$

Contraceptive agents are prescribed, but often without licence, in adolescents for a wide range of non-contraceptive indications such as treatment of delayed puberty 
and menstrual disorders (irregular periods, dysmenorrhoea). ${ }^{27}$ Whatever their application, safety and efficacy should be determined in appropriate clinical trials in relevant age groups. ${ }^{2}$ This is especially important in adolescence when pharmacokinetics, therapeutic effects, side effects and future impact of any complications of medications can rarely be accurately obtained from studies in adults or in different species.

Neither ethical nor legal considerations provide valid reasons to exclude 13-15 year olds from trials of contraceptive agents, subject to appropriate ethical approval. Such approval can be granted if there is a sound scientific basis, a defined clinical need and prospect of benefit to the individual or the class they represent, provided that there is minimal risk and minimal burden. ${ }^{1}$ Advances in understanding of adolescent brain development have provided greater insight into both adolescent risk-taking behaviours and their capacity to assimilate and process information relating to research trials. ${ }^{28}$ Moreover, the capacity of 14 year olds to provide informed consent for research or treatment is indistinguishable from that of adults. ${ }^{29}$ But, in contrast to provision of contraceptive advice or treatment (which can occur without parental consent or knowledge for those who are Gillick competent ${ }^{16}$ ), participation of under $16 \mathrm{~s}$ in CTIMPs requires consent of a legally designated representative (usually a parent) as well as the subject's agreement, ${ }^{2}$ even though the latter's refusal will be honoured. This potential requirement for parental consent poses practical difficulties for the conduct of contraceptive trials in under 16 s since adolescents value strongly the right to confidentiality in sexual health matters $^{31}$ and are owed the same duty of confidentiality as adults. ${ }^{18}$

Trial designs will need to overcome this potential problem as well as recognising the possibility that 13 -year old to 15 -year-old participants could be subject to abuse, exploitation or coercion, from which they need safeguarding. Though there is no mandatory statutory requirement to report sexual activity in this age group in the UK, there is strong presumption that reasonable suspicions of abuse or exploitation will be discussed with designated or named child safeguarding professionals and an appropriate strategy agreed and documented. ${ }^{32-34}$ Clinical trials units undertaking such research must have protocols in place, reviewed and accepted by local safeguarding professionals, that deal with potential disclosure of child sexual abuse, positive
Box 2 Suggested guidance for

research ethics committees

reviewing studies of contraceptive

agents in children < 16 years of age

- Neither ethical nor legal considerations provide valid reasons to exclude 13-15 year olds from trials of contraceptive agents, subject to appropriate ethical approval.

- Any clinical trials unit undertaking such research must have protocols in place that have been reviewed and accepted by local designated or named professionals for safeguarding children who deal with any disclosure of child sexual abuse, positive pregnancy testing or other safeguarding concern.

- Staff of CTUs responsible for recruiting children for such research should have the necessary competencies to enable them to detect and act upon child safeguarding concerns in accordance with protocols.

- While contraceptive advice/treatment can occur without parental consent for those under 16 years of age who are Gillick competent, clinical trials of investigational medicinal products participation requires consent from a legally designated representative (usually parent) as well as subject agreement.

- Trials involving contraceptive agents in those $<13$ years of age in the UK, perhaps with the intention global licensing, are more difficult for research ethics committees to consider. Advice from the Health Research Authority National Research Ethics Advisors' Panel should be sought.

pregnancy testing or other safeguarding concern. They should be able to reassure RECs that those who will be responsible for recruiting young people to trials will have appropriate training and competencies to enable them to detect safeguarding concerns and to act upon them in accordance with agreed protocols. ${ }^{32}$

Trials that involve testing of contraceptive agents in children under the age of 13 years in the UK, perhaps with the intention of licensing for wider global usage, are more difficult for RECs. Despite the protection to health professionals afforded by the SOA, it remains problematic for UK
RECs to sanction trials of agents that permit an activity to which the young person cannot legally consent. Presumably, RECs could sanction trials of agents that had an acceptable primary therapeutic purpose, for example, control of menstrual conditions, even if there was a secondary (unintended?) effect of contraception. We would recommend that all such trials should be discussed with the Health Research Authority National Research Advisors Panel before any favourable REC decision enables the trial to proceed.

Those faced with granting ethical approval of trials of contraception in children $<16$ years of age need to be satisfied that such trials are both scientifically justified and lawful and that appropriate mechanisms to respect confidentiality and provide safeguards for participants are in place.

Based on our analysis, we suggest simple guidance for those RECs tasked with the review of contraceptive agent investigative medical product studies in children $<16$ years of age (box 2 ).

Competing interests None.

Provenance and peer review Not commissioned; externally peer reviewed.
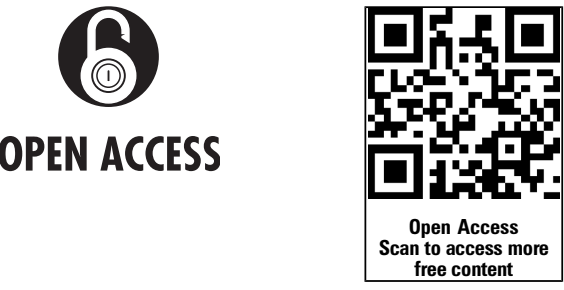

Open Access This is an Open Access article distributed in accordance with the Creative Commons Attribution Non Commercial (CC BY-NC 4.0) license, which permits others to distribute, remix, adapt, build upon this work non-commercially, and license their derivative works on different terms, provided the original work is properly cited and the use is noncommercial. See: http://creativecommons.org/licenses/ by-nc/4.0/

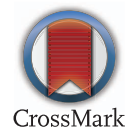

To cite Brierley J, Larcher V. Arch Dis Child 2014;99:1070-1073.

Received 28 May 2014

Revised 9 August 2014

Accepted 13 August 2014

Published Online First 4 September 2014

Arch Dis Child 2014;99:1070-1073.

doi:10.1136/archdischild-2014-306854

\section{REFERENCES}

1 WMA Declaration of Helsinki-Ethical Principles for Medical Research Involving Human Subjects 2013. http://www.wma.net/en/30publications/10policies/b3/ (accessed May 2014). 
2 Directive 2001/20/Ec Of The European Parliament And Of The Council of 4 April 2001 on the approximation of the laws, regulations and administrative provisions of the Member States relating to the implementation of good clinical practice in the conduct of clinical trials on medicinal products for human use. http://www.eortc.be/ services/doc/clinical-eu-directive-04-april-01.pdf (accessed May 2014)

3 Office for National Statistics Statistics Bulletin. Conceptions in England and Wales. 2012. http:// www.ons.gov.uk/ons/dcp171778_353922.pdf (accessed May 2014).

4 Faculty of Sexual \& Reproductive Healthcare Clinical Guidance Contraceptive Choices for Young People Clinical Effectiveness Unit March 2010. http://www. fsrh.org/pdfs/ceuGuidanceYoungPeople2010.pdf (accessed May 2014).

5 Larcher V. Developing guidance for checking pregnancy status in adolescent girls before surgical, radiological or other procedures. Arch Dis Child 2012;97:857-60.

6 Whincup PH, Gilg JA, Odoki K, et al. Age of menarche in contemporary British teenagers: survey of girls born between 1982 and 1986. BMJ 2001;322:1095-6.

7 Wellings K, Nanchalal K, MacDowall W, et al. Sexual behaviour in Britain: early heterosexual experiences. Lancet 2001;358:1843-50.

8 National Survey of Sexual Attitudes and Lifestyles 1990 and 2000. http://www.natcen.ac.uk/natsal (accessed Dec 2012).

9 Gupta N, Corrado S, Goldstein M. Hormonal contraception for the adolescent. Pediatr Rev 2008;29:386-97.

10 Deans E, Grimes D. Intrauterine devices for adolescents: a systematic review. Contraception 2009;79:418-23.

11 Harper CC, Stratton L, Raine TR, et al. Counseling and provision of long-acting reversible contraception in the US: national survey of nurse practitioners. Prev Med 2013:57:883-8.
12 Bennett SE, Assefi NP. School-based teenage pregnancy prevention programs: a systematic review of randomized controlled trials. J Adolesc Health 2005:36:72-81.

13 Jemmott JB, Jemmott LS, Fong GT. Efficacy of a theory-based abstinence-only intervention over 24 months a randomized controlled trial with young adolescents. Arch Pediatr Adolesc Med 2010;164:152-9

14 Arie $S$. Has Britain solved its teenage pregnancy problem? BMJ 2014;348:g256115.

15 Blake SM, Ledsky R, Goodenow C, et al. Condom availability programs in Massachusetts high schools; relationships with condom use and sexual behaviour. American J Public Health 2003:91:955-62.

16 Gillick v West Norfolk \& Wisbech AHA \& DHSS [1985] 3 WLR (HL).

17 Age of Legal Capacity (Scotland) Act 1991. http:// www.legislation.gov.uk/ukpga/1991/50/contents (accessed May 2014).

18 General Medical Council 0-18 years: guidance for all doctors. GMC London 2007. http://www.gmc-uk.org/ guidance/ethical_guidance/children_guidance_index. asp (accessed May 2014).

19 Sexual Offences 1985. Act 2003. http://www. legislation.gov.uk/ukpga/2003/42 (accessed May 2014).

20 Wheeler R, Kohler J, Paget J, et al. Sperm preservation in children: a prescription for consistency with both child-protection and the criminal law. Clinical Risk 2011;17:15-18.

21 Schedule 1 to the Medicines for Human Use (Clinical Trials) Regulations 2004 (S.I.2004:1031) as amended by S.I.2006:1928, S.I.2006:2984 and S.I.2008:941.

22 European Clinical Trials Directive (EC2001/20).

23 UK Medical Eligibility Criteria for Contraceptive use UKMEC 2009. http://www.fsrh.org/pdfs/ UKMEC2009.pdf (accessed May 2014).

24 Centers for Disease Control and Prevention. United States medical eligibility criteria for contraceptive use. MMWR 2010:59:1-6.
25 National institute for Healthcare and Excellence. Contraceptive services with a focus on young people up to the age of 25. Issued: March 2014 NICE public health guidance 51. http://www.nice.org.uk/nicemedia/ live/14476/67004/67004.pdf (accessed May 2014).

26 National institute for Healthcare and Excellence. Long-acting reversible contraception. Issued: October 2005 last modified: April 2013 NICE clinical guideline 30. http://www.nice.org.uk/nicemedia/live/ 10974/29909/29909.pdf7 (accessed May 2014).

27 Fraser I, Kovacs $\mathrm{G}$. The efficacy of non-contraceptive uses for hormonal contraceptives. Med J Aust 2003:178:621-3.

28 Burke TM, Abramovitch R, Zlotkin S. Children's understanding of the risks and benefit associated with research. J Med Ethics 2005;31: 715-20.

29 Kuther T, Posada M. Children and adolescents' capacity to provide informed consent for participation in research. Adv Psychol Res 2004;32:164-73.

30 Weithorn LA, Campbell SB. The competency of children and adolescents to make informed treatment decisions. Child Dev 1982;53:1585-8.

31 McPherson A. Adolescents in primary care. $\mathrm{Br} \mathrm{Med} \mathrm{J}$ 2006;330:465-7.

32 Safeguarding Children and Young People-Roles and Competencies for healthcare staff-Intercollegiate document. shttp://www.saphna-professionals.org/ files/docs/feguarding_Children_and_Young_people_ 2010_1_.pdf (accessed May 2014).

33 Working Together to Safeguard Children. HM Government 2010.5.27-5.30. http://webarchive. nationalarchives.gov.uk/20130401151715/https:// www.education.gov.uk/publications/ eorderingdownload/00305-2010dom-en-v3.pdf (accessed May 2014)

34 Working Together to Safeguard Children. HM Government 2013. https://www.gov.uk/government/ uploads/system/uploads/attachment_data/file/ 281368/Working_together_to_safeguard_children. pdf (accessed May 2014). 